\title{
Status of the New Muonium HFS Experiment at J-PARC/MUSE
}

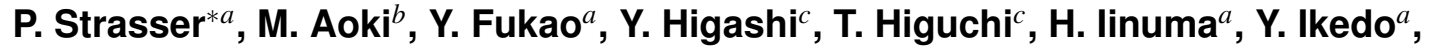 \\ K. Ishida $^{d}$, M. Iwasaki ${ }^{d}$, R. Kadono ${ }^{a}$, O. Kamigaito ${ }^{d}$, S. Kanda ${ }^{e, d}$, D. Kawall ${ }^{f}$, \\ N. Kawamura ${ }^{a}$, A. Koda ${ }^{a}$, K. M. Kojima ${ }^{a}$, K. Kubo ${ }^{g}$, Y. Matsuda ${ }^{c}$, T. Mibe ${ }^{a}$, Y. Miyake ${ }^{a}$, \\ T. Mizutani ${ }^{c}$, K. Nagamine ${ }^{a}$, K. Nishiyama ${ }^{a}$, T. Ogitsu ${ }^{a}$, R. Okubo ${ }^{a}$, N. Saito ${ }^{a}$, \\ K. Sasaki ${ }^{a}$, K. Shimomura ${ }^{a}$, M. Sugano ${ }^{a}$, M. Tajima ${ }^{c}$, K. S. Tanaka ${ }^{c, d}$, D. Tomono ${ }^{h}$, \\ H. A. Torii ${ }^{c}$, E. Torikai ${ }^{i}$, A. Toyoda ${ }^{a}$, K. Ueno ${ }^{a}$, Y. Ueno ${ }^{c}$, A. Yamamoto ${ }^{a}$, and \\ M. Yoshida ${ }^{a}$ \\ ${ }^{a} \mathrm{KEK}$ \\ 1-1 Oho, Tsukuba, Ibaraki 305-0801, Japan \\ ${ }^{b}$ Osaka University \\ Toyonaka, Osaka 560-0043, Japan \\ ${ }^{c}$ Graduate School of Arts and Sciences, University of Tokyo \\ 3-8-1 Komaba, Meguro-ku, Tokyo 153-8902, Japan \\ ${ }^{d}$ RIKEN \\ 2-1 Hirosawa, Wako, Saitama 351-0198, Japan \\ ${ }^{e}$ Graduate School of Science, University of Tokyo \\ 7-3-1 Hongo, Bunkyo-ku, Tokyo 113-0033, Japan \\ ${ }^{f}$ University of Massachusetts \\ Amherst, USA \\ ${ }^{g}$ International Christian University (ICU) \\ Mitaka, Tokyo 181-8585, Japan \\ ${ }^{h}$ Graduate School of Science, Kyoto University \\ Sakyo-ku, Kyoto 606-8501, Japan \\ ${ }^{i}$ University of Yamanashi \\ Kofu, Yamanashi 400-8511, Japan
}

At the J-PARC Muon Science Facility (MUSE), the MuSEUM ${ }^{\dagger}$ collaboration is aiming at an improved measurement of the ground state hyperfine structure (HFS) of muonium [1]. High precision measurement of muonium HFS is the most sensitive tool for testing QED theory and measuring fundamental constants of the muon magnetic moment and mass, which are currently determined by the previous experiment at LAMPF [2]. An overview of the different aspects of this new muonium HFS measurement, and the current status of the preparation are presented.

16th International Workshop on Neutrino Factories and Future Neutrino Beam Facilities - NUFACT2014, 25 -30 August, 2014

University of Glasgow, United Kingdom

\footnotetext{
* Speaker on behalf of the MuSEUM Collaboration.

†The collaboration name MuSEUM stands for "Muonium Spectroscopy Experiment Using Microwave".
} 


\section{Introduction}

Muonium (Mu) is a leptonic system that is made of a bound state of a positive muon with an electron. It is also regarded as a light isotope of hydrogen, with the feature that neither of its constituents have an internal structure, and therefore more adequate than hydrogen for precision spectroscopy because uncertainty on the proton radius prohibits precise comparison between theory and measurements. High precision measurements of muonium ground state hyperfine structure (HFS) can be regarded as the most sensitive tool for testing quantum electrodynamics (QED) theory, and for determining fundamental constants of the muon magnetic moment $\left(\mu_{\mu}\right)$ and hence its mass $\left(m_{\mu} \propto \mu_{\mu}^{-1}\right)$. Those values are currently determined by the previous muonium HFS experiment at LAMPF [2] performed already more than 15 years ago. The present accuracy is $12 \mathrm{ppb}$ for the muonium ground state hyperfine transition frequency $(\Delta v)$ and $120 \mathrm{ppb}$ for the ratio of the magnetic moments between muon and proton $\left(\mu_{\mu} / \mu_{p}\right)$, respectively.

Since muonium is a pure leptonic atom, the value of the HFS transition frequency is calculated with high precision using QED theory. However, at the moment the theoretical accuracy is only 63 ppb [3]. This could be improved if higher-order terms were included in the QED calculation. It is said that theorists are confident that muonium HFS can be calculated to $10 \mathrm{~Hz}$ (about $2 \mathrm{ppb}$ ) within a few years, if needed. Thus, the necessity of a more precise measurement of the muonium HFS to test even further QED theory.

A more precise determination of $\mu_{\mu}$ would contribute to improve the precision of the new muon g-2 measurements planned at J-PARC [4] and also at Fermilab [5]. Muon g-2 is regarded as a keystone at unraveling the physics beyond the standard model. The value of $\mu_{\mu} / \mu_{p}$ is required in the determination of the muon anomalous magnetic moment $a_{\mu}\left(\equiv \frac{g-2}{2}\right)$, which is also called "muon g-2". This is due to the fact that proton NMR is used for field measurement. It accounts to approximately $30 \mathrm{ppb}$ to the present measured precision of $540 \mathrm{ppb}$ of the muon anomalous magnetic moment. The goal of the new muon g-2 measurements is to reach a precision of $100 \mathrm{ppb}$, thus new high precision muonium HFS spectroscopy results would be beneficial.

Another motivation is the implication of a possible test of CPT and Lorentz invariance from microwave spectroscopy of muonium [6]. CPT and Lorentz violation incorporated in extensions to the standard model lead to potentially observable perturbation in the ground state energy levels of muonium. Lagrangian terms beyond the standard model, which violate CPT and Lorentz invariance, would contribute shifts in muonium HFS frequencies. The nonstandard theory indicates that muonium HFS frequencies should oscillate with the earth's sidereal frequency and that those frequencies would be anticorrelated in the laboratory frame. Yet another important reason to improve the precision of muonium HFS measurements.

The experimental uncertainty on the muonium HFS in the previous LAMPF experiment was mainly dominated by statistical error (about $90 \%$ ). The rest were systematic uncertainty that included inhomogeneity in the magnetic field, muon stopping distribution, microwave power and gas density extrapolation. Our goal is to improve the precision of the previous measurement by one order of magnitude. The new high intensity surface muon beam that will soon be available at J-PARC/MUSE H-Line [7] will provide an opportunity to achieve that goal. An overview of the different aspects of this new muonium HFS measurement is presented. 


\section{Experimental Approach}

The Breit-Rabi energy level diagram for muonium atom is shown in Fig. 1. The ground state hyperfine splitting $\Delta v$ is to be measured by a microwave magnetic resonance technique. This transition is not measured directly at zero magnetic field but following the approach of the previous experiment [2] at a field of $1.7 \mathrm{~T}$. The static magnetic field due to the Zeeman effect and the applied external RF field splits the ground state of muonium into four different substates and the two spinflip resonance frequencies $v_{12}$ and $v_{34}$ are measured. The sum of the two transition frequencies $v_{12}$ and $v_{34}$ is constant independently of the applied static magnetic field, and is equal to the ground state hyperfine splitting at zero field, i.e., $\Delta v=v_{12}+v_{34}$. Also, the difference between those two transition frequencies is directly related to the ratio of the magnetic moments of muon and proton, i.e., $\mu_{\mu} / \mu_{p} \propto v_{34}-v_{12}$. Therefore, the sum of the measured frequencies gives the QED test, while the difference is used to determine the values of the muon magnetic moment and mass.

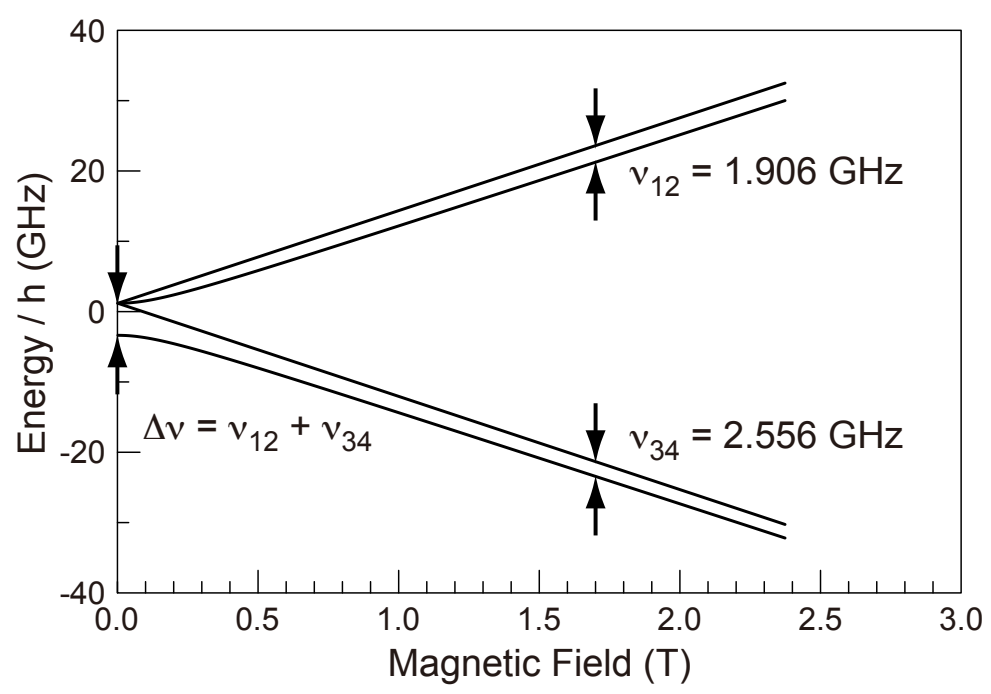

Figure 1: Breit-Rabi energy level diagram for muonium atom.

Figure 2 shows the schematic view of the experimental setup. The experimental procedure can be summarized as follows: (1) muonium formation, (2) RF spin flip, and (3) positron asymmetry measurement. The high intensity surface muon beam with an expected intensity of $1 \times 10^{8} \mu^{+} / \mathrm{s}$ will be provided by the new H-Line that is under construction at J-PARC/MUSE [7]. The muon beam has a double-pulsed structure of $100 \mathrm{~ns}$ wide, separated by $600 \mathrm{~ns}$ with a repetition rate of 25 Hz. The positive muons $\left(\mu^{+}\right)$are $100 \%$ backward polarized with respect of the muon momentum direction and injected into a large superconducting solenoid with an applied static field of $1.7 \mathrm{~T}$. At the center of the solenoid, a RF cavity is located inside a gas chamber containing highly pure krypton gas. The profile of the incident muon beam is measured by an online, non-destructive, beam profile monitor located in front of the entrance window of the gas chamber. The $\mu^{+}$are stopped in the $\mathrm{Kr}$ gas volume and form polarized muonium atom through the charge exchange reaction $\mu^{+}+\mathrm{Kr} \rightarrow \mathrm{Mu}+\mathrm{Kr}^{+}$. The muon spin can be flipped by applying a microwave magnetic 
field in the RF cavity perpendicular to the static magnetic field of the solenoid. The static magnetic field and introduced RF field splits the ground state of the muonium into four different substates. The RF cavity was designed to have two frequency modes, TM110 and TM210, to match the resonance frequencies $v_{12}$ and $v_{34}$, respectively. The positrons $\left(\mathrm{e}^{+}\right)$from muon decay are emitted preferentially in the direction of the muon spin. At the resonance, the RF field induces the muon spin flip changing the angular distribution of the emitted positrons from primarily backward to forward direction. Positrons are detected with segmented scintillation detectors placed downstream and upstream of the gas chamber. Muonium spectroscopy is then performed by scanning the RF frequency and measuring the positron asymmetry to determine both resonance frequencies $v_{12}$ and $v_{34}$, respectively.

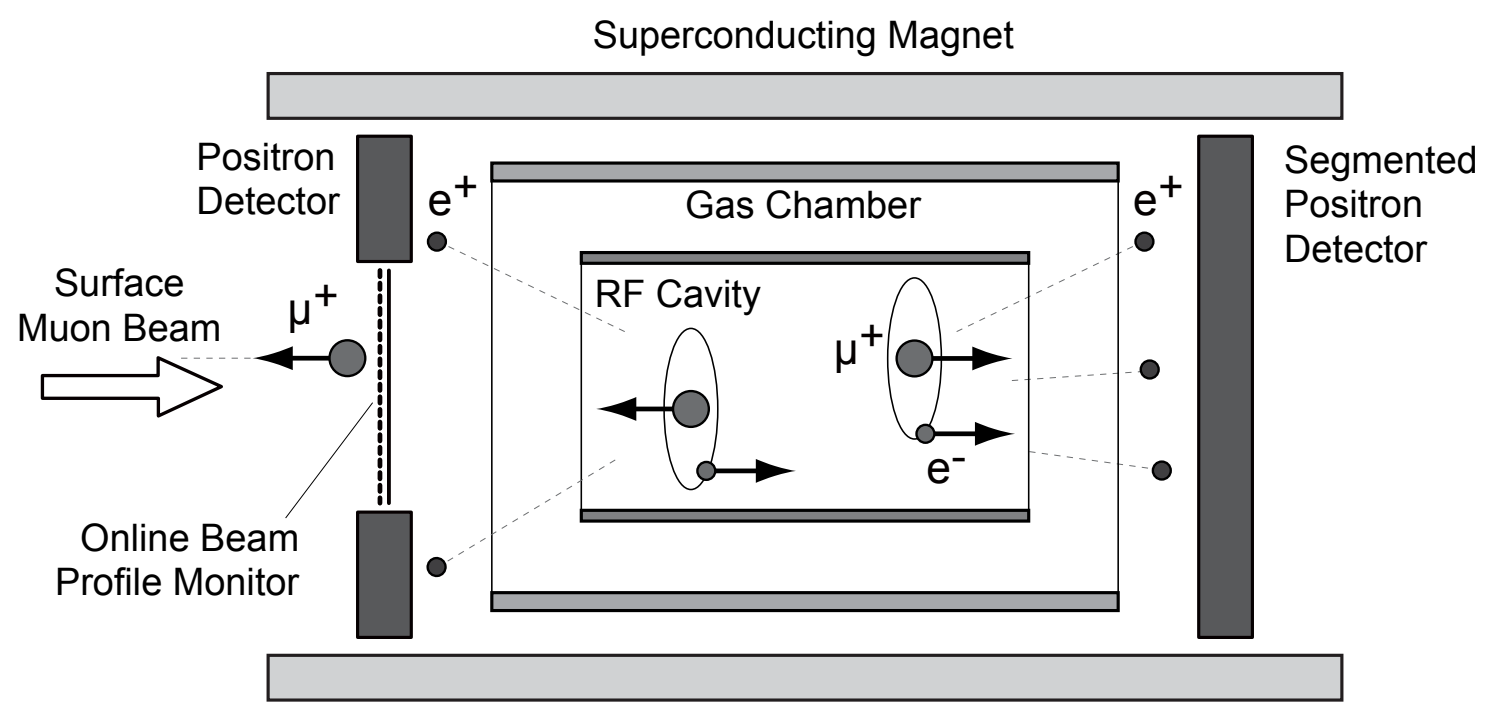

Figure 2: Schematic view of the experimental setup.

\section{Current Status}

An overview of the different aspects of this new muonium HFS measurement, including recent developments and the current status of the preparation of the gas chamber, RF cavity, integrated detector system and magnetic field measurement system are presented.

\subsection{Target chamber and RF system}

The target gas chamber and the RF cavity are now constructed. The cavity is made of oxygenfree copper with windows $25-\mu \mathrm{m}$ thick to let muons in. The cavity length is $304 \mathrm{~mm}$, in comparison to $160 \mathrm{~mm}$ for the LAMPF experiment, to allow measurement at lower $\mathrm{Kr}$ gas density, thus reducing the systematic uncertainty on the gas density extrapolation to the zero density limit. The gas pressure can be changed from 0.3 to $1.5 \mathrm{~atm}$. The use of a longer cavity was required to stop muons in lower gas density and also to reduce systematic uncertainty due to muon stopping distribution. 
A simulation showed that even at the lowest density, $94 \%$ of the muons will stop in the cavity. The RF cavity is tuned by displacing alumina tuning rods. Consistency checks were successfully performed to assess the proper manufacturing of the cavity and the resonance tuning system with simulations. Further details on the RF system development are reported in [8]. Since resonance lines are distorted by fluctuating RF power, coaxial pipes and a feedback loop from a pickup antenna in the cavity to monitor and regulate the power will be used to achieve a RF power stability of $0.1 \%$. The error from the RF power is below $2 \mathrm{~Hz}$ when compared to the mesured frequency. The RF system and feedback circuit will be assembled and tested in 2015.

The target chamber is made of pure aluminum with $100-\mu$ m thick windows to contain the $\mathrm{Kr}$ gas. The gas pressure will be monitored by a silicon pressure transducer at a level of $2 \times 10^{-5}$. Gas samples will be collected in small cylinders at given intervals during the measurement to monitor the gas purity to ppm level or below using a Q-mass spectrometer. A gas handling system has been fabricated to perform the necessary gas manipulation. A preliminary test performed at the end of 2014 without the precise pressure detector and the sampling cylinders was conclusive. In 2015, the precise gas pressure transducer will be mounted on the gas handling system, and pressure stability and purity measurements will be performed.

The climate control of the experimental area is very important to reduce fluctuation on the gas pressure and RF system. The temperature variation in the experimental area will be kept below 1 degree.

\subsection{Beam profile monitor}

The profile monitor is required to be non-destructive as much as possible and to measure the muon beam pulse by pulse to reject irregular beam shape and pulse intensity. A new profile monitor using plastic scintillation fiber with SiPM (Silicon PhotoMultiplier) is being developed. The use of plastic scintillation fiber as compared to plastic scintillator slab allows to minimize the light reflection loss and have a better optical coupling to the SiPM. Also, there is less position dependence along the fiber and possibly better control of the effective thickness. The profile monitor has a total detection area of $100 \mathrm{~mm} \times 100 \mathrm{~mm}$, and each axis comprises 16 segments. One segment consists of an array of 40 plastic scintillation fiber $100-\mu \mathrm{m}$ in diameter, i.e., 4-mm effective width, bound together in a bundle and directly connected to a SiPM at both ends [9]. Fibers are bounded on a polyimide film $25-\mu \mathrm{m}$ thick with epoxy resin, each segment spaced from the next with a 2-mm gap. The front-end electronics is composed of an EASIROC [10] chip that is used as an ASD (Amplifier Shaper Discriminator) and peak-hold ADC (Analog-to-Digital Converter). A prototype comprising two segments was constructed and tested at the end of 2014. The test results are promising and the data are now being analyzed. The fiber bounding process will be optimized to get a uniform thickness. Based on the final results the design of the full-scale detector will be finalized and constructed in 2015.

\subsection{Positron detector}

Positrons are detected with highly segmented scintillation counters to have high-rate capability due to the pulse nature of the muon beam. The total detection area is $300 \mathrm{~mm} \times 300 \mathrm{~mm}$, divided into 900 segments. Each segment has two SiPM's to require a coincidence hit in order to suppress accidental background due to dark noise. We currently use Hamamatsu MPPC (Multi-Pixel 
Photon Counter) as SiPM. Signals from the MPPC's are directly processed by KALLIOPE (KEK Advanced Linear and Logic board Integrated Optical detector for Positron and Electron) readout circuit [11]. This circuit contains an ASIC-based (Application Specific Integrated Circuit) ASD and FPGA-based (Field Programmable Gated Arrays) multi-hit TDC (Time-to-Digital Converter). A prototype of 9 segments was successful developed as proof of concept. The development of this integrated detector system is described in details in Ref. [9, 12]. The detector pileup was carefully studied. From a simulation, the maximum expected event rate is approximately $3 \mathrm{MHz}$, resulting in a pileup loss of $2 \%$ of the total statistics. The pileup loss is dominated by the analog pulse shape, which depends on the amplifier parameters. Optimization of these parameters to minimize the systematic uncertainty is being investigated.

\subsection{Offline muon beam monitor}

The muon stopping distribution in the $\mathrm{Kr}$ gas chamber will be measured by an offline muon beam monitor. This monitor is based on a new muon beam profile monitor that was developed to diagnose pulsed muon beams at J-PARC/MUSE [13]. It is mainly composed of a scintillation screen, a gated image intensifier, and a cooled CCD camera. The scintillation screen is placed inside the gas chamber, without the RF cavity installed, closed with an acrylic plate. The scintillation light from the screen is capture by a large aperture lens and sent to the image-intensified CCD unit. By moving the scintillation screen together with the detector unit, i.e. keeping the focal length of the lens constant, the muon stopping distribution can be measured along the gas chamber. This offline muon beam monitor was tested in a beamtime at the end of 2014, and muon stopping distributions were successfully measured at several Kr gas density.

\subsection{Magnetic field}

The superconducting solenoid that will be used is an old MRI magnet, which was designed to provide high homogeneity magnetic field. The main specifications of the magnet are an effective bore diameter of $680 \mathrm{~mm}$, a field inhomogeneity of less than $1 \mathrm{ppm}$ in a spheroid of R300 $\mathrm{mm} \times$ Z200 mm, and a stability of $0.03 \mathrm{ppm} /$ hour. Our choice of a longer cavity to allow measurement at lower $\mathrm{Kr}$ gas density imposes strict requirements on the magnet. We aim at a field homogeneity of less than $1 \mathrm{ppm}$ with absolute calibration in a spheroidal volume of $\phi 200 \mathrm{~mm} \times 300 \mathrm{~mm}$ (muon stopping region). A precise field monitoring system using a set of water NMR probes to scan the spheroidal region ( 24 probes $\times 24$ positions) is under development. A pulsed NMR technique is used in measuring the magnetic field. A preliminary measurement test showed that a magnetic field accuracy of $33 \mathrm{ppb}$ can be achieved. The fine adjustment of the magnetic field homogeneity will be carried out by shim coils and the insertion of iron shim plates. Shim plate positioning will be calculated using a singular value decomposition method; an iterative process of field correction and precise field measurement. The prototype test of the NMR probe with a precision of better than $100 \mathrm{ppb}$ is scheduled in winter of 2014/2015. Also, investigation of the diamagnetic correction of the NMR probe by a FEM numerical simulation and measurement of the probe shape effect is in progress. 


\section{Estimation of experimental accuracy}

As already mentioned earlier, the uncertainty in the previous experiment at LAMPF was mainly dominated at $90 \%$ by statistical error. Sources of systematic uncertainty included in order of importance inhomogeneity in the magnetic field, microwave power, muon stopping distribution, and gas density extrapolation. The H-Line at J-PARC/MUSE with $1 \times 10^{8} \mu^{+} / \mathrm{s}$ and considering a measurement time of 100 days would give a total muon number of $1 \times 10^{15}$, hundred times more statistics than the previous experiment, thus ten times reduction of the statistical uncertainty. But with better statistics, the systematic uncertainty becomes more severe and needs to be carefully considered. It should be noted that understanding of the systematical error in an experiment is often limited by the measurement time. A tool to estimate systematic uncertainty in the experiment from several sources was developed [14, 15], and is currently being used to investigate the effect a particular fluctuation on the final result. Here are a few considerations:

(1) The microwave power has a direct impact on the resonance intensity. Any power fluctuation or drift during the resonance scan would deteriorate the measured resolution. We need to measure the center of the resonance line to a level of a few hertz. Using that simulation tool, it was found that a fluctuation of $0.1 \%$ in the RF power results in a shift of $10 \mathrm{~Hz}$ in the measured frequency, thus contributing $2.0 \mathrm{ppb}$ to $\delta(\Delta v)$ and $20 \mathrm{ppb}$ to $\delta\left(\mu_{\mu} / \mu_{p}\right)$, respectively. It was also verified that a position drift of the muon beam distribution during the scan affects the measured resolution, thus the need of a constant monitoring the muon beam profile.

(2) The magnetic field measurement accuracy has little effect on the determination of $\Delta v$, but is very important in $\mu_{\mu} / \mu_{p}$. The present accuracy of $30 \mathrm{ppb}$ would account for $15 \mathrm{ppb}$ in $\delta\left(\mu_{\mu} / \mu_{p}\right)$. (3) The beam profile will be monitored online for every beam pulse with a precision of $1 \mathrm{~mm}$. A 1mm error would result in a shift of $3 \mathrm{~Hz}$, or $0.6 \mathrm{ppb}$ and $6 \mathrm{ppb}$ for $\delta(\Delta v)$ and $\delta\left(\mu_{\mu} / \mu_{p}\right)$, respectively. Also, the longitudinal muon stopping distribution will be measured to an equal precision of $1 \mathrm{~mm}$ by the offline muon beam monitor. Here, the effect is less severe and would only account in a shift of $<1 \mathrm{~Hz}$, or $0.2 \mathrm{ppb}$ and $2 \mathrm{ppb}$, respectively.

(4) The quadratic dependence of the gas density extrapolation to the zero density limit will be improved by our ability to measure at lower $\mathrm{Kr}$ gas density with a longer cavity. The systematic uncertainty would be reduced to a shift of $5 \mathrm{~Hz}$, thus contributing $1.0 \mathrm{ppb}$ and $5 \mathrm{ppb}$, respectively.

After considering all other error sources, the tentative error budget for systematic uncertainty in our experiment is at present estimated to $\sim 2.4 \mathrm{ppb}$ for $\delta(\Delta v)$ and $\sim 27 \mathrm{ppb}$ for $\delta\left(\mu_{\mu} / \mu_{p}\right)$, respectively.

\section{Summary}

The improvement of the muonium HFS measurement is important for further QED testing and coming new fundamental physics experiments. Ameliorating the overall accuracy, estimating and understanding systematic uncertainties are essential in reaching that goal. Development of integrated detector system, field monitoring system and RF system are at the focus of the preparation for the experiment. At present, it is unclear when exactly the new H-Line will be operational, but we are aiming at being ready as soon as the first muon beam will be available, maybe at the end of 2015 or early 2016. 


\section{References}

[1] K. Shimomura et al., Possibility of precise measurements of muonium HFS at J-PARC MUSE, AIP Conf. Proc. 1382 (2011) 245.

[2] W. Liu et al., High Precision Measurements of the Ground State Hyperfine Structure Interval of Muonium and of the Muon Magnetic Moment, Phys. Rev. Lett. 82 (1999) 711.

[3] D. Nomura and T. Teubner, Hadronic contributions to the anomalous magnetic moment of the electron and the hyperfine splitting of muonium, Nucl. Phys. B 867 (2013) 236.

[4] K. Ishida et al., Muon g-2/EDM at J-PARC, in proceedings of 16th International Workshop on Neutrino Factories and Future Neutrino Beam Facilities (NUFACT2014), to be published in Proceedings of Science PoS(NUFACT2014)098 (2015).

[5] J. M. Grange et al., g-2 at Fermilab, in proceedings of 16th International Workshop on Neutrino Factories and Future Neutrino Beam Facilities (NUFACT2014), to be published in Proceedings of Science PoS(NUFACT2014)099 (2015).

[6] V. W. Hughes et al., Test of CPT and Lorentz Invariance from Muonium Spectroscopy, in Hydrogen Atom: Precision Physics of Simple Atomic Systems, S. G. Karshenboim et al. (Eds.), Lecture Notes in Physics 570, Springer, Berlin, 2001, p. 397-406.

[7] N. Kawamura et al., H line; a beam line for fundamental physics study, J. Phys.: Conf. Ser. 551 (2014) 012062.

[8] K. S. Tanaka et al., Measurement of muonium hyperfine splitting at J-PARC, in proceedings of International Conference on Exotic Atoms and Related Topics (EXA2014), to be published in Hyperfine Interactions (2015).

[9] S. Kanda et al., Precision measurement of muonium hyperfine splitting at J-PARC and integrated detector system for high-intensity pulsed muon beam experiment, in proceedings of 2nd International Symposium on Science at J-PARC (J-PARC 2014), to be published in JPS Conference Proceedings (2015).

[10] S. Callier et al., EASIROC, an Easy \& Versatile ReadOut Device for SiPM, Phys. Procedia 37 (2012) 1569.

[11] K. M. Kojima et al., New MSR spectrometer at J-PARC MUSE based on Kalliope detectors, J. Phys.: Conf. Ser. 551 (2014) 012063.

[12] S. Kanda et al., Development of High-Rate Positron Tracker for the Muonium Production Experiment at J-PARC, JPS Conf. Proc. 2 (2014) 010404.

[13] T. U. Ito et al., Online full two-dimensional imaging of pulsed muon beams at J-PARC MUSE using a gated image intensifier, Nucl. Instr. Meth. A 754 (2014) 1.

[14] K. S. Tanaka et al., Measurement of Muonium Hyperfine Splitting at J-PARC, JPS Conf. Proc. 2 (2014) 010405.

[15] H. Torii et al., Precise Measurement of Muonium HFS at J-PARC MUSE, in proceedings of 2nd International Symposium on Science at J-PARC (J-PARC 2014), to be published in JPS Conference Proceedings (2015). 